\section{Compliance and viewpoint of glaucoma patients in Greece}

\begin{abstract}
Purpose To document the prevalence of non-compliance and to investigate patients' perceptions concerning glaucoma in a Greek cohort.

Methods We investigated 100 consecutive patients referred to our glaucoma clinic and already treated for chronic glaucoma. Compliance and patients' insight were ascertained by two independent observers by means of a predetermined questionnaire. All patients were subsequently assessed for their ability to instil their eyedrops accurately.

Results Fifty one per cent of our patients were not aware of the nature of glaucoma, but $80 \%$ were afraid it might lead to blindness. Clinically significant non-compliance (more than two doses missed per week) was established in $44 \%$ of our patients. Men and those using eyedrops more than 4 times a day were more likely to default. Non-compliant patients exhibited higher mean intraocular pressure (22.9 vs $18.5 \mathrm{mmHg} ; p>0.001)$ and worse visual field loss $(10.8$ vs $7.0 \mathrm{~dB}$; $p=0.008$ ) compared with compliant patients. Involuntary non-compliance was also common in our group, with only $53 \%$ instilling their eye drops accurately.
\end{abstract}

A.G.P. Konstas

G. Maskaleris

S. Gratsonidis

C. Sardelli

Department of

Ophthalmology

Aristotle University of

Thessaloniki

Greece

A.G.P. Konstas, MD, PhD

(Glasg)

University Department of

Ophthalmology

AHEPA Hospital

Kiriakidi 1

Thessaloniki, Greece

Tel: + 31994752

Fax: +31286487

e-mail:

konstas@med.auth.gr

Supported by a grant from KESY

Received: 16 September 1999 Accepted in revised form:

13 April 2000
A.G.P. KONSTAS, G. MASKALERIS,

S. GRATSONIDIS, C. SARDELLI predict accurately the behaviour of the glaucoma patient and is often unable to distinguish between the compliant and noncompliant patient. ${ }^{8}$ Furthermore, the problem may be compounded if the ophthalmologist mistakes non-compliance for ineffectiveness of a given glaucoma medication and prescribes more potent medications. ${ }^{7}$ Overall, there is a growing awareness that there is a high prevalence of undiagnosed non-compliance in elderly patients with glaucoma, with significant repercussions for their management. ${ }^{9-11}$ To explain the reasons why patients do not take their antiglaucoma medications as prescribed it is vital to consider their viewpoint of glaucoma. ${ }^{12}$

Glaucoma is a major contributor to blindness in Greece. ${ }^{13,14}$ The present study was established firstly to document the Greek patients' viewpoint of glaucoma and secondly to determine the extent and significance of noncompliance in a cohort of glaucoma patients.

\section{Materials and methods}

This prospective study was set up to provide data on the compliance and viewpoint of Greek glaucoma patients receiving topical treatment with eyedrops. The study recruited 100 consecutive patients with chronic open angle glaucoma referred to our glaucoma clinic and already receiving antiglaucoma medications. Our university hospital provides secondary and tertiary care for a population of approximately 1.5 million people. Between January 1996 and July 1997100 consecutive patients with either primary open angle glaucoma $(n=52)$ or exfoliation glaucoma $(n=48)$ were enrolled into this study, which was conducted in the University Department of Ophthalmology, AHEPA Hospital, Thessaloniki, Greece. Exfoliation glaucoma and primary open angle glaucoma patients were chosen because they comprise the vast majority of glaucoma patients in Greece. ${ }^{13}$ Patients included in the present study were previously diagnosed and treated for glaucoma by state insurance or private ophthalmologists. However, the patients recruited to this study were not selected (i.e. were not 'problem patients') since the majority of glaucoma patients in our area are insured in 
the state insurance scheme (IKA) and are routinely co-managed with their ophthalmologist, who refers them for confirmation of the diagnosis and visual fields. Furthermore, many patients book an appointment in our glaucoma clinic to obtain a hospital prescription which allows them to obtain their antiglaucoma medications almost free of charge.

Excluded from this study were patients with other types of glaucoma such as congenital, closed angle, or other secondary glaucomas (pigmentary, etc.). Patients with other ophthalmic conditions, or those who underwent surgery (factors that might influence the management of glaucoma), were also excluded. All patients recruited demonstrated typical glaucomatous visual field loss and glaucomatous optic nerve head cupping with open, normal-appearing angles.

Patients were first interviewed by two observers (S.G. and C.S.) who at the time of the study were medical students. An open questionnaire strategy was used and all patients were informed that the interview was confidential. The questions to assess the patients' viewpoint of glaucoma included the following: How did you find out that you had glaucoma? Did your ophthalmologist provide adequate information concerning the disease? What do you know about glaucoma? What do you fear most about glaucoma? Can you name your eyedrops and how often in a day you have to take them? Patients were also asked whether they had any problems with their medications and if so whether these had influenced their administration. Compliance with antiglaucoma therapy was investigated in all patients during this interview by asking how often they miss a dose of their medication and the reason, if any, for missing doses. Emphasis was also placed on whether they had encountered side effects in the past and, if so, whether this influenced their use of eyedrops.

Following the interview all patients underwent a comprehensive evaluation of their glaucoma by two glaucoma specialists (A.G.P.K. and G.M.) who were masked to the questionnaire results. Glaucoma data from all patients were stored in a standard protocol.

Information documented on all patients included: sex, age, systemic disorders, family history of glaucoma and glaucoma data from the time of diagnosis to the present. All concomitant systemic disorders (e.g. arthritis) that might adversely influence the use of drops were recorded. Patients were divided into three categories depending on the prescribed frequency of instillation of their eyedrops (up to twice a day, up to four times a day, and more than four times a day). Ocular evaluation included Snellen visual acuity, Goldmann applanation tonometry, examination of the ocular adnexa, slit-lamp biomicroscopy, gonioscopy and stereoscopic evaluation of the fundus with the Volk 90 D lens. Glaucomatous visual field loss was determined by automated static threshold perimetry (Octopus $500 \mathrm{EZ}$; peridata 6.2c). To identify unintentional non-compliance (termed dyscompliance) all patients were tested for their manual dexterity in compressing the drug bottles and their ability to instil the drops accurately. Data from this assessment were inserted in the patient protocols and were used to determine the rate of dyscompliance. Patients were divided into three categories: 'successful', if they were capable of targeting the drop accurately; 'moderately successful', if they had to try more than once and eventually managed to instil sufficient quantity of their medication, or if they relied on someone else to successfully instil their drops; and 'unsuccessful' (clinically significant problem), if they missed the eye more than $50 \%$ of the time due to poor manual dexterity and targeting.

Following this assessment, all patients were given detailed instruction on how to comply with their medication regimes. In many cases the same treatment regime obtained a significant reduction in intraocular pressure. Patients who could not, or were not able to, comply were offered another management option (laser treatment or surgery).

Statistical analyses were performed with the Excel 5.0 software package. Student's $t$-test, the chi-square analysis and Fisher's exact test were used where appropriate. The significance level was set at $5 \%$.

\section{Results}

At the time of the interview the average age of the patients was 65.4 years (range $43-82$ years). There were 47 men and 53 women. In our cohort only 9 patients (9\%) had been diagnosed as a result of glaucoma screening (due to a positive family history and other risk factors). Some patients were diagnosed by a random eye check (39\%) and others because of perceived visual disturbances $(35 \%)$, or on account of ill-defined complaints (17\%). In 42 patients the prescribed daily frequency of glaucoma medications was up to two times a day (beta-blockers or dorzolamide), in 20 patients up to four times a day (beta-blockers combined with dipivefrine or dorzolamide) and in 38 patients more than four times a day (beta-blockers with pilocarpine and other combinations).

To assess the viewpoint of our patients regarding the nature of glaucoma we defined as the 'correct' answer 'a disorder with increased pressure in the eye commensurate with optic nerve damage'. Although a definition of glaucoma that includes raised intraocular pressure may not be appropriate nowadays amongst glaucoma specialists, this simplification was felt necessary for our patients. This question elicited, in descending order, that $51 \%$ of our patients did not know what glaucoma is; that $27 \%$ knew only that glaucoma is a disease that leads to blindness; and finally, that only $22 \%$ had a relatively accurate idea about the nature of glaucoma (20 knew it was pressure elevation in the eye, and 2 knew it included nerve damage due to pressure). Sixty-five per cent of our patients claimed that at the time of diagnosis their ophthalmologist did not provide adequate information concerning their disease. The majority of our patients stated that what they fear most about having glaucoma is blindness $(80 \%)$; 18 patients feared nothing and 2 feared they might need surgery. 
During the interview 64 patients could not accurately identify the name of their medications and 14 could not remember the exact dosing regime. With regard to problems perceived by patients with the daily use of medications, these (in descending order) were: irritation/itching $(56 \%)$, redness $(5 \%)$, blurring of vision (5\%). Thirty-four patients $(34 \%)$ did not report any problem with their medications.

The assessment of drug compliance revealed that unsatisfactory compliance with medications was common: of the 100 patients interviewed only 56 stated categorically that they never miss a dose. Fifteen patients reported that they only missed a dose 1-3 times a month. A clinically significant problem with non-compliance (deemed as more than two doses missed per week) was identified in 29 patients who stated that they missed more than 2 doses every week $(15 \%)$, or missed more than half their doses (11\%). Finally 3 patients $(3 \%)$ were not using their medications at all. Reasons quoted for defaulting were (in descending order) lack of visual symptoms without treatment and/or blurring of vision with medication $(34 \%)$, forgetfulness $(28 \%)$, medication unavailable $(15 \%)$, inconvenient frequency $(16 \%)$ and absence of someone to instil the drops (5\%).

Of equal importance was the assessment of unintentional non-compliance (termed dyscompliance). Only 53 of the 100 patients tested were judged very capable of instilling their drops accurately. Thirty-two patients $(32 \%)$ were moderately successful either by trying more than once and eventually instilling sufficient a quantity of their medication, or by relying on someone else to instil their eyedrops. Fifteen patients, however, had a clinically significant problem, missing the eye more than $50 \%$ of the time due to poor manual dexterity and targeting. Thirteen of these patients were responsible for administering their own eyedrops and 2 had a relative to assist them. Of these 15 unintentional non-compliers there were 4 elderly individuals $(4 \%)$ for whom dyscompliance was due to physical inability to use their antiglaucoma bottles properly due to systemic conditions (arthritis, tremor). None of the other patients had a significant problem with systemic diseases or, if they did, their drops were instilled successfully by a relative. Therefore in the present study the rate of clinically significant non-compliance was $44 \%$ (29\% voluntary and $15 \%$ involuntary non-compliance).

These 44 non-compliant patients showed higher mean intraocular pressure $(22.9 \pm 3.7 \mathrm{mmHg})$ compared with the compliant patients $(18.6 \pm 3.5 \mathrm{mmHg} ; p>0.001)$. They also exhibited more disc cupping $(0.69 \pm 0.1$ vs $0.62 \pm 0.1 ; p=0.017)$ and worse mean visual field loss $(10.8 \pm 5.8$ vs $7.0 \pm 5.4 \mathrm{~dB} ; p=0.008)$ compared with compliant patients. There was no difference in the mean visual acuity of the two groups $(0.83 \pm 0.2$ vs $0.89 \pm 0.1$; $p=0.195)$.

When comparing the prescribed frequency of instillation with the reported compliance there was a significant difference between non-compliance in the glaucoma group using eyedrops up to two times a day (5 of 42 patients; $12 \%$ ) compared with the glaucoma group prescribed eyedrops more than four times a day (16 of 38 patients; $42 \%)(p<0.05)$. Furthermore, in our glaucoma cohort non-compliance was found to be more prevalent in men $(21$ of $47 ; 45 \%$ ) than women ( 8 of $53 ; 15 \%$ ) $(p<0.05)$.

No relationship was found between non-compliance and type of glaucoma (exfoliation glaucoma or primary open angle glaucoma), age, frequency of side effects to medications (indeed side effects were reported most commonly amongst those who were compliant). We were unable to compare the effect of patients' knowledge about glaucoma with the frequency of non-compliance because the vast majority of our patients $(78 \%)$ did not have an accurate viewpoint of the nature of glaucoma.

\section{Discussion}

This is the first study investigating the rate of noncompliance in a Greek glaucoma cohort. Our results support previous research ${ }^{6,10,11}$ which suggests that noncompliance is a major contributor to glaucoma blindness. Between $28 \%$ and $58 \%$ of glaucoma patients do not use their medications as prescribed. ${ }^{3,6,8,10,15}$ Even this may be a conservative estimate because the true extent of noncompliance remains unknown in many cohorts ${ }^{16}$ and our compliance data may be partially flawed. The latter point was illustrated by Novack et al. ${ }^{17}$ who found that the participation of glaucoma patients in a study investigating compliance may improve their compliance by up to $30 \%$. Importantly, our study did not encompass the rate of a patient's participation in his or her health care (e.g. in keeping hospital appointments or seeking medical advice) and thus may underestimate noncompliance.

In our study the most common reason for noncompliance was the perceived lack of visual symptoms with glaucoma. Thus, in our cohort non-compliance was associated with lack of insight into the nature of glaucoma. First Riffenburgh ${ }^{18}$ has stated that glaucoma is the most important area where the patient's viewpoint can alter the results. Poor understanding of the disease and its treatment, as seen in our study cohort, may lead to a higher degree of non-compliance. ${ }^{19}$ Vincent $^{20}$ reported that compliant patients were more likely to know that glaucoma is associated with increased intraocular pressure. In another study, ${ }^{3}$ which like ours used the interview technique, non-compliant patients with glaucoma were inadequately aware of the association between glaucoma and blindness. In general medicine it has been shown that medication compliance can improve by up to $36 \%$ by intensive individual teaching of patients in the clinic and at home. ${ }^{21}$ In another randomised clinical trial employing a medication monitor, Norell ${ }^{22}$ found that education significantly improved compliance.

The results of our study demonstrate that in Greece there is a need for continuing patient education of glaucoma patients. It is well established in the literature that poor communication between patient and clinician leads to more defaulting. ${ }^{3,16,19}$ Parkin et al. ${ }^{19}$ found that 
46 of the 66 non-compliant patients in their study did not have a clear understanding of their regimen. It is clear from the available literature ${ }^{1,6,7}$ that better doctor-patient communication may improve a patient's knowledge of glaucoma and facilitate better compliance.

Differing criteria for defining non-compliance make it difficult to compare the various studies. For instance Vincent ${ }^{20}$ reported a rate of $58 \%$ for non-compliance by using the criterion of more than one missed dose of medication per month. Using that criterion most of our patients would have been classified as defaulters. We decided to arbitrarily define 'clinically significant noncompliance' as more than two doses missed per week, as we felt this figure would be more relevant to clinical practice. It is to be emphasised that this definition is not ideal since missing two doses would be of far greater significance to a patient who only uses a beta-blocker once a day than to a patient who uses eight drops a day.

There are limitations in studies such as ours which attempt to establish the rate of non-compliance using hospital interviews. Our cohort is a selected clinic-based population. There is often underreporting of missed doses by an interview ${ }^{5}$ and estimates of non-compliance based on reports by patients may be inaccurate. ${ }^{11,23}$

Electronic monitors may provide more accurate data, but as illustrated by Novack et al. ${ }^{17}$ patients participating in a study investigating compliance with monitors may show a better than 'real world' compliance by up to $30 \%$. In a study that attempted to side-step direct information on compliance Rotchford and Murphy ${ }^{11}$ calculated in 55 patients how much timolol was dispensed relative to their need based on their regimen. They found that 51\% of their patients had insufficient drops dispensed to comply with treatment as prescribed, and calculated for non-compliant patients a mean period of 85 days per year without drops.

In the light of the results from the present study it is clear that a significant proportion of our patients unintentionally default from the prescribed treatment. Unintentional non-compliance, or dyscompliance, was an unexpected but clinically significant problem in our group which cannot be solved by the prescription of more medications and more frequent dosing. Increased vigilance is required to identify patients who cannot instil their drops properly. As in the study by Winfield et al. ${ }^{24}$ our study has identified dyscompliance as a significant component of non-compliance. It is probably worthwhile for ophthalmologists to ask their patients to demonstrate how they instil their eyedrops and to emphasise proper application techniques. Further studies are necessary to assess the scale of dyscompliance amongst glaucoma patients since this component of noncompliance should be more amenable to therapeutic intervention (teaching, drops instilled by a relative).

Our study has found that non-compliant patients had higher mean treated intraocular pressure and worse visual field loss. Although these findings show a noteworthy association it is by no means possible to demonstrate a causal relationship from a study such as ours. Further research is required to determine the impact of non-compliance in the rate of glaucomatous visual loss.

Non-compliance may be of greater importance in a high-pressure glaucoma such as exfoliation glaucoma ( $48 \%$ of our cohort) for which it is likely that the worse intraocular pressure characteristics account for the more rapid visual deterioration in comparison with primary open angle glaucoma. ${ }^{25-30}$ Furthermore, exfoliation glaucoma patients often require treatment with more antiglaucoma drops. ${ }^{13,25}$ It is therefore crucial to identify non-compliers with hypertensive glaucomas and to consider prompt surgical intervention.

The impressions of previous workers ${ }^{10}$ that noncompliance may be more common in patients receiving more medications is validated in the present study. We found a correlation between the rate of non-compliance and the frequency of administration of antiglaucoma medications. Currently, the availability of new, welltolerated glaucoma medications (e.g. latanoprost, brimonidine, Cosopt) may offer a wider range of therapeutic choices and, most importantly, require less frequent administration. Keeping glaucoma regimens as simple as possible, using single-eyedrop regimens and longer-acting antiglaucoma eyedrops may diminish the rate of non-compliance. ${ }^{7,31}$ Further research is required to validate this. There is also much scope for further research in the success of patient education in reducing clinically significant non-compliance and thus glaucoma blindness. Such data are important to determine whether glaucoma patients' behaviour can be meaningfully influenced, since appropriate treatment strategies can be implemented either way (i.e. teaching or earlier surgery for non-compliers) to prevent visual loss.

We are grateful to Professor G. N. Dutton for his help with this manuscript.

\section{References}

1. Goldberg I. Compliance. In: Ritch R, Shields MB, Krupin T, editors. The glaucomas. St Louis: Mosby, 1996:1375-84.

2. Zimmermann T, Zalta A. Facilitating patient compliance in glaucoma therapy. Surv Ophthalmol Suppl 1983;28:252-7.

3. Block S, Rosenthal AR, Friedman L, Caldarolla P. Patient compliance in glaucoma. Br J Ophthalmol 1977;61:531-4.

4. Ashburn F, Goldberg I, Kass M. Compliance with ocular therapy. Surv Ophthalmol 1980;24:237-46.

5. Norell S. Accuracy of patient interviews and estimates by clinical staff in determining medication compliance. Soc Sci Med 1981;15:37-61.

6. Kass MA. Compliance and prognosis in glaucoma. Arch Ophthalmol 1985;103:504.

7. van Buskirk E. The compliance factor. Am J Ophthalmol 1986;105:609-10.

8. Kass MA, Meltzer DW, Gordon M, et al. Compliance with topical pilocarpine treatment. Am J Ophthalmol 1986;101:515-23.

9. MacKean J, Elkington A. Compliance with treatment of patients with chronic open-angle glaucoma. Br J Ophthalmol 1983;67:46-9.

10. Patel SC, Spaeth GL. Compliance in patients prescribed eyedrops for glaucoma. Ophthalmic Surg 1995;26:233-6.

11. Rotchford AP, Murphy KM. Compliance with timolol treatment in glaucoma. Eye 1998;12:234-6. 
12. Marston M. Compliance with medical regimens: a review of the literature. Nurs Res 1970;19:312-23.

13. Konstas AGP. Morphological and clinical studies on the exfoliation syndrome and open angle glaucoma. PhD Thesis, Glasgow, 1993:1-364.

14. Konstas AGP, Tsatsos I, Kardasopoulos T, Bufidis T, Maskaleris G. Preoperative features of patients with exfoliation glaucoma and primary open-angle glaucoma. The AHEPA study. Acta Ophthalmol Scand 1998;76:208-12.

15. Granstrom PA. Glaucoma patients not compliant with their drug therapy: clinical and behavioural aspects. Br J Ophthalmol 1982;66:467-70.

16. Davis M. Variations in patients' compliance with doctors: analysis of congruence between survey responses and results of empirical investigations. J Med Educ 1966;41:1037-48.

17. Novack GD, Davod R, Lee PF, et al. Effects of changing medication regimes in glaucoma patients. Ophthalmologica 1988;196:23-8.

18. Riffenburgh R. Doctor-patient relationship in glaucoma therapy. Arch Ophthalmol 1966;75:204-6.

19. Parkin D, Hanney C, Quirk J, Crooks J. Deviation from prescribed drug treatment after discharge from hospital. BMJ 1976;II:686-8.

20. Vincent P. Factors influencing patient noncompliance: a theoretical approach. Nurs Res 1971;20:509-16.

21. Hecht A. Improving medication compliance by teaching outpatients. Nurs Forum 1974;13:113-29.

22. Norell S. Improving medication compliance: a randomised clinical trial. BMJ 1979;II:1031-3.
23. Gordis L, Markowitz M, Lilienfeld A. The inaccuracy in using interviews to estimate patient reliability in taking medications at home. Med Care 1969;7:49-54.

24. Winfield AJ, Jessiman D, Williams A, Esakowitz L. A study of the causes of non-compliance by patients prescribed eyedrops. Br J Ophthalmol 1990;74:477-80.

25. Konstas AGP, Stewart WC, Stroman GA, Sine CS. Clinical presentation and initial treatment patterns in patients with exfoliation glaucoma versus primary open-angle glaucoma. Ophthalmic Surg Lasers 1997;28:111-7.

26. Granstrom P. Progression of visual field defects in glaucoma: relation to compliance with pilocarpine therapy. Arch Ophthalmol 1985;103:529-31.

27. Konstas AGP, Mantziris DA, Stewart WC. Comparison of diurnal variation in newly diagnosed untreated patients with exfoliation glaucoma and primary open angle glaucoma. Arch Ophthalmol 1997;115:182-5.

28. Konstas AGP, Mantziris D'A, Cate EA, Stewart WC. Effect of timolol on the diurnal intraocular pressure in exfoliation and primary open-angle glaucoma. Arch Ophthalmol 1997;115:975-9.

29. Lindblom B, Thorburn W. Functional damage at diagnosis of primary open angle glaucoma. Acta Ophthalmol 1984;62:223-9.

30. Pohjanpelto P. Long-term prognosis of visual field in glaucoma simplex and glaucoma capsulare. Acta Ophthalmol 1985;63:418-23.

31. Davidson S, Akingbenin T. Compliance in ophthalmology. Trans Ophthalmol Soc UK 1980;100:286-9. 